\title{
Design of effectively single-mode leakage channel fibers with large mode area and low bending loss
}

\author{
Kunimasa Saitoh $^{\mathrm{a})}$, Yukihiro Tsuchida, and Masanori Koshiba \\ Graduate School of Information Science and Technology, Hokkaido University, \\ Sapporo 060-0814, Japan \\ a)ksaitoh@ist.hokudai.ac.jp
}

\begin{abstract}
Novel design of large-mode-area leakage channel fibers (LCFs) with a single air-hole ring surrounding a multi-unit-cell solid core is investigated for realizing robust single-mode and low bending loss characteristics. We perform detailed numerical simulations using a finite element method, and we show that the proposed LCFs with a solid core formed by multiple missing neighboring air-holes can achieve much lower bending losses compared with the reported LCF with six large air-holes.
\end{abstract}

Keywords: optical fiber, leakage channel fiber, large mode area Classification: Optical fiber

\section{References}

[1] Y. Jeong, J. K. Sahu, D. N. Payne, and J. Nilsson, "Ytterbium-doped large-core fibre laser with $1 \mathrm{~kW}$ of continuous-wave output power," Electron. Lett., vol. 40, pp. 470-472, April 2004.

[2] A. Galvanauskas, "Mode-scalable fiber-based chirped pulse amplification systems," IEEE J. Sel. Top. Quantum Electron., vol. 7, pp. 504-517, July/Aug. 2001.

[3] M. D. Nielsen, J. R. Folkenberg, and N. A. Mortensen, "Singlemode photonic crystal fibre with effective area of $600 \mu \mathrm{m}^{2}$ and low bending loss," Electron. Lett., vol. 39, pp. 1802-1803, Dec. 2003.

[4] L. Dong, X. Peng, and J. Li, "Leakage channel optical fibers with large effective area," J. Opt. Soc. Am. B, vol. 24, pp. 1689-1697, Aug. 2007.

[5] K. Saitoh and M. Koshiba, "Full-vectorial imaginary-distance beam propagation method based on finite element scheme: Application to photonic crystal fibers," IEEE J. Quantum Electron., vol. 38, pp. 927-933, July 2002.

[6] K. Kakihara, N. Kono, K. Saitoh, and M. Koshiba, "Full-vectorial finite element method in a cylindrical coordinate system for loss analysis of photonic wire bends," Opt. Exp., vol. 14, pp. 11128-11141, Nov. 2006.

[7] K. Saitoh, Y. Tsuchida, M. Koshiba, and N. A. Mortensen, "Endlessly single-mode holey fibers: the influence of core design," Opt. Exp., vol. 13, pp. 10833-10893, Dec. 2005. 


\section{Introduction}

Large-mode-area (LMA) single-mode optical fibers have attracted much attention regarding application of high-power fiber lasers [1]. The power levels in the range of a few tens of watts to multi-kilowatts have been realized and further power level enhancement of fiber lasers is strongly demanded for industrial purposes. The main problem in the development of higher output power is the nonlinear effects, such as self-phase modulation, Raman scattering, and Brillouin scattering, in both continuous wave and mode-locked fiber lasers. The nonlinear effects can be partly overcome by using LMA optical fibers with reduced numerical aperture (NA), which can maintain the single-mode operation, however, the reduction of NA leads to a significant increase of macro-bending loss. An effectively single-mode operation can be achieved for practical application by precisely designed optical fiber that supports few modes with higher NA. However, the increment of the core size is limited up to $30 \mu \mathrm{m}$ due to the difficulty in single-mode operation [2]. Even if we use all-silica photonic crystal fibers (PCFs) which allow very broad-band single-mode LMA, it is also limited to a core size of about $30 \mu \mathrm{m}$ by excessive bending losses [3].

Perhaps, one of the most impressive and promising approach to realize LMA optical fiber with robust single-mode operation is the use of leakage channel fibers (LCFs), where a single air-hole ring forms a core, reported by Dong et al. [4]. The reported LCF core is formed by six large air-holes and precisely designed to create large leakage loss for higher-order modes (HOMs), while maintaining negligible leakage loss for the fundamental mode. However, from the theoretical point of view, the LCF design with leakage loss of the second-order mode of larger than $1 \mathrm{~dB} / \mathrm{m}$ and that of the fundamental mode of less than $0.1 \mathrm{~dB} / \mathrm{m}$ reported in Ref. [4] can not provide sufficiently low bending loss characteristics. In this work, we propose and numerically investigate a novel design of single-mode LCF with LMA and low bending loss in which the microstructured optical fiber is formed by a single air-hole ring as in the reported LCF, while the solid core region is formed by the removing multiple air-holes. We perform detailed numerical simulations using a modal solver based on the finite element method (FEM) [5, 6], and we show that the proposed LCFs with a solid core formed by multiple missing neighboring air-holes can achieve much lower bending losses compared with the reported LCF with six large air-holes [4].

\section{Leakage Channel Fiber Design}

Figure 1 (a) shows the cross section of a conventional LCF design proposed by Dong et al. [4], where the fiber core is formed by six air holes (shown as blue circles) and a solid core region is created by 1-unit-cell area (denoted from now on as LCF1). The background material is pure silica, the airhole diameter is $d$, and the center-to-center hole spacing is $\Lambda$. Contrary to the standard step-index fiber, all modes in a microstructured optical fiber with finite number of air-hole rings are inherently leaky because the core 


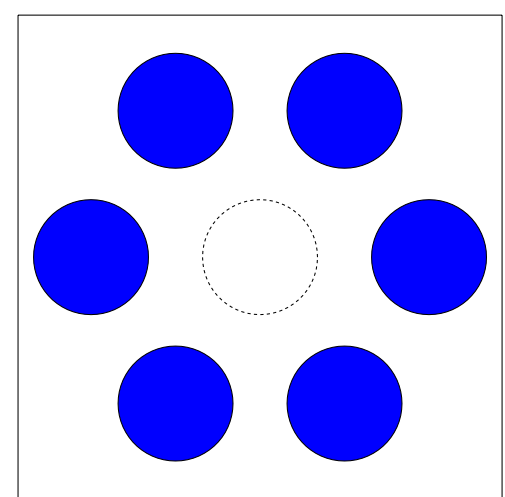

(a) LCF1

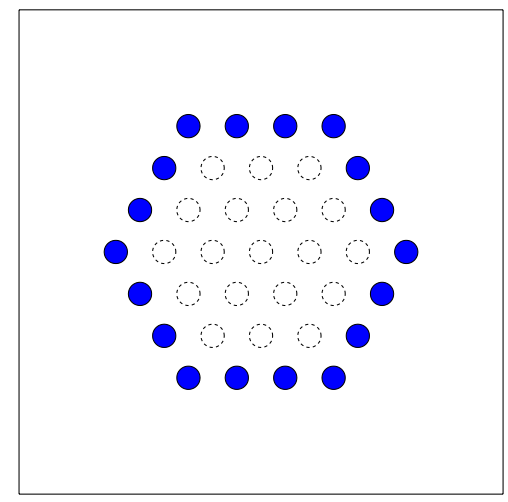

(c) LCF19

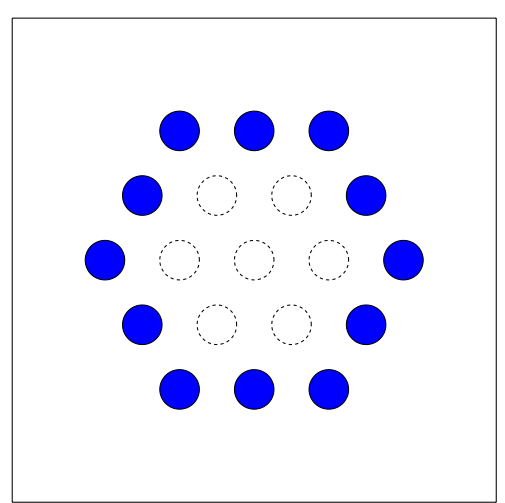

(b) LCF7

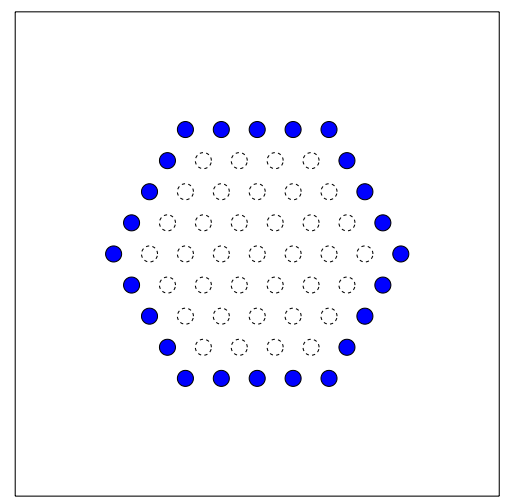

(d) LCF37

Fig. 1. Schematic cross-sections of leakage channel fibers formed by (a) 6 air holes with a single rod in core (LCF1), (b) 12 air holes with 7 neighboring rods in core (LCF7), (c) 18 air holes with 19 neighboring rods in core (LCF19), and (d) 24 air holes with 37 neighboring rods in core (LCF37). The blue circle represents air hole and the dashed circle denotes the silica region.

index is the same as the index of the outer cladding region without air holes. This leaky nature of all modes in a LCF enables engineering of differential propagation loss of modes [4], which can not be realized in conventional optical fibers. Figures 1 (b)-(d) show the cross sections of newly proposed LCF designs. The dashed circle in Fig. 1 denotes the silica region and has been shown to clarify the core design. The LCF in Fig. 1 (b) with a single airhole ring is formed by 12 air holes and a core region is created by 7 -unit-cell area (LCF7). If the core sizes of LCF1 and LCF7 are same, the hole-pitch $\Lambda$ and the hole-diameter $d$ in LCF7 have to be small compared to those in LCF1 in order to achieve effectively single-mode operation [7]. Similarly, the LCFs in Figs. 1 (c) and (d) are formed by 18 air holes with 19-unit-cell area in core (LCF19) and 24 air holes with 37-unit-cell area in core (LCF37), respectively.

\section{Numerical results}

Through an accurate modal analysis performed using a full-vector FEM solver [5], we evaluate the effective mode area, $A_{\text {eff }}$, and leakage loss varia- 
tions as a function of the hole pitch, $\Lambda$, and the normalized air-hole diameter, $d / \Lambda$. Figures $2(\mathrm{a}),(\mathrm{b}),(\mathrm{c})$, and (d) show the contour plots of the effective mode area (color maps) of the fundamental mode, the degenerated $\mathrm{HE}_{11}$ mode, at 1064-nm operating wavelength mapped into the range of the design parameters $\Lambda$ and $d / \Lambda$, in four different LCF designs, LCF1, LCF7, LCF19, and LCF37, respectively, where the refractive index of silica is assumed to be 1.45 and the air index is 1 . The solid black curve depicted inside the graph corresponds to the leakage loss value of $1 \mathrm{~dB} / \mathrm{m}$ at $1064 \mathrm{~nm}$ for the second group of the HOMs, namely, $\mathrm{TE}_{01}$ mode, $\mathrm{TM}_{01}$ mode, and the degenerated $\mathrm{HE}_{21}$ mode. Since we are considering a LCF for high-power ytterbium fiber lasers in a device length of $1-2 \mathrm{~m}$, we assume that $1 \mathrm{~dB} / \mathrm{m}$ is the minimum leakage loss for the suppression of second-order HOMs in their propagation [4]. Therefore, the solid curve corresponds to the upper limit of the structural parameters. In addition, we assume that the maximum leakage loss limit of the fundamental mode is $0.3 \mathrm{~dB} / \mathrm{m}$ in order to ensure negligible fundamental transmission loss. The dashed black curve, shown in Fig. 2, corresponds to the leakage loss value of $0.3 \mathrm{~dB} / \mathrm{m}$ at $1064 \mathrm{~nm}$ for the fundamental mode, also represents the lower limit of the structural parameters. If we use a LCF near the upper boundary (solid curve), a lower leakage loss for the fundamental mode is expected, while near the lower boundary (dashed curve), a higher leakage loss for the HOM is expected. From these results we can deduce effectively single-mode LCF structures with specific core size.

Considering a target effective mode area value of $1400 \mu \mathrm{m}^{2}$ (i.e. a core

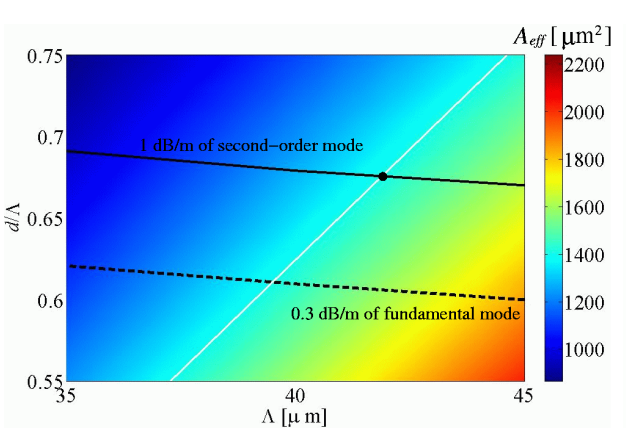

(a) LCF1

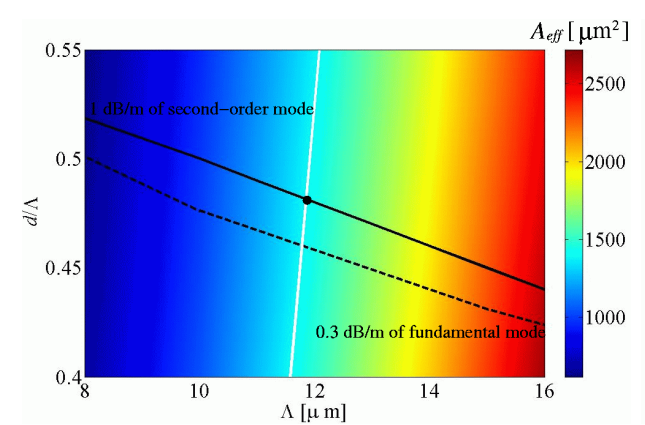

(c) LCF19

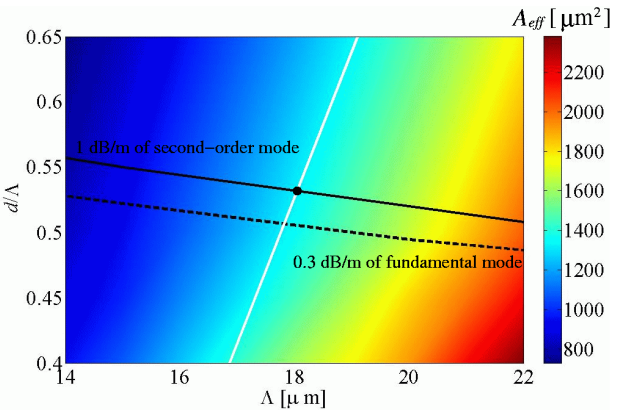

(b) LCF7

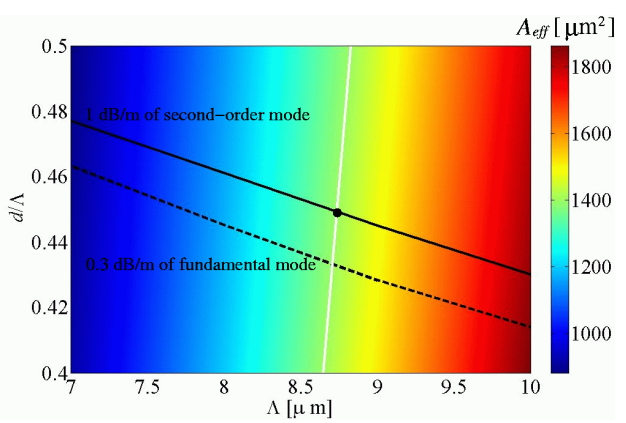

(d) LCF37

Fig. 2. Effective mode area of the fundamental mode at 1064-nm operating wavelength mapped into the range of the design parameters of $\Lambda$ and $d / \Lambda$ for (a) LCF1, (b) LCF7, (c) LCF19, and (d) LCF37. 


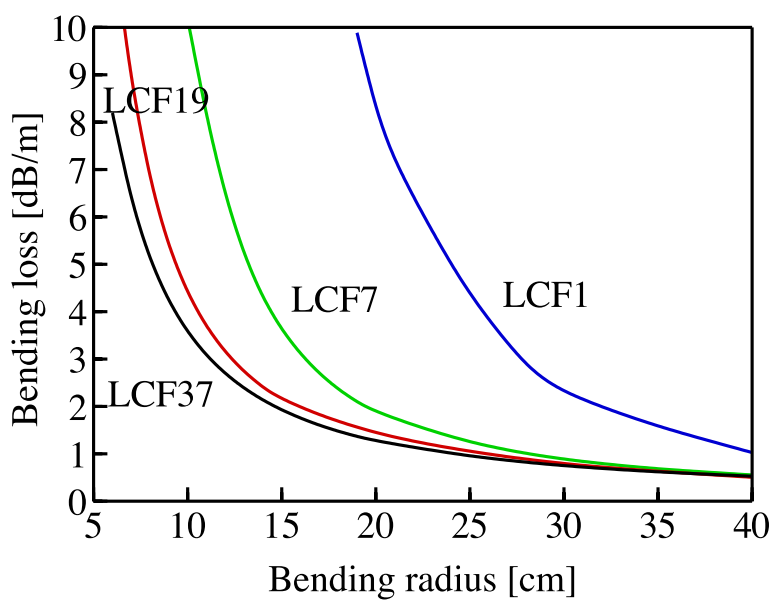

(a)

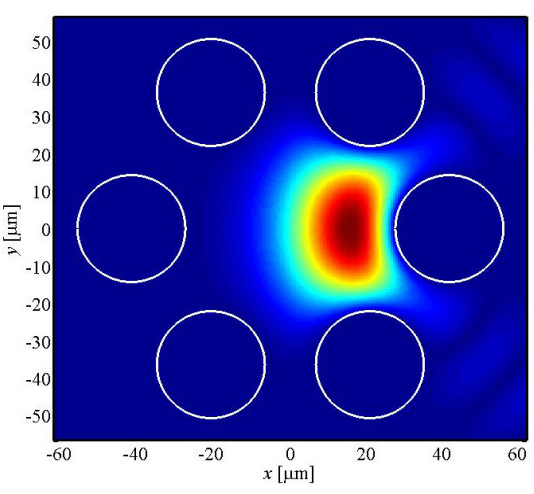

(b)

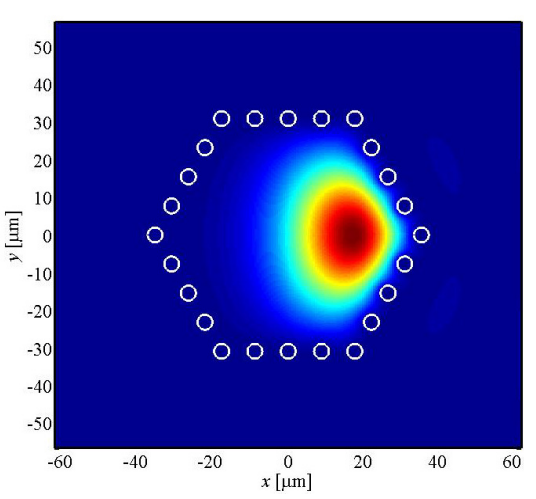

(c)

Fig. 3. (a) Bending losses in $\mathrm{dB} / \mathrm{m}$ as a function of the bending radius in $\mathrm{cm}$ at 1064-nm wavelength. (b) The optical field distributions of the fundamental mode at a wavelength of $1064 \mathrm{~nm}$ in curved LCF1 $(\Lambda=41.9 \mu \mathrm{m}, d / \Lambda=0.68)$ and $(\mathrm{c}) \operatorname{LCF} 37(\Lambda=$ $8.9 \mu \mathrm{m}, d / \Lambda=0.446)$, where the bending radius is $15 \mathrm{~cm}$.

diameter of $\sim 50 \mu \mathrm{m}$ ) at 1064-nm wavelength, we depict the white solid curve corresponding the effective mode area value of $1400 \mu \mathrm{m}^{2}$ in Fig. 2. In Fig. 2 (a), the crossing point of the white and black solid curves (upper limit) corresponds to the structural parameters of $\Lambda=41.9 \mu \mathrm{m}$ and $d / \Lambda=0.68$, while the white and dashed curves cross at $\Lambda=39.5 \mu \mathrm{m}$ and $d / \Lambda=0.61$ (lower limit). We have confirmed from a bending loss point of view that the LCF at upper limit (corresponding to the black filled circle in Fig. 2) has lower bending loss performance compared to the LCF at lower limit due to lesser leakage loss for the fundamental mode. Therefore, we focus the LCF structures at upper limit for the following bending loss evaluation. The structural parameters at upper limit are $\Lambda=18.5 \mu \mathrm{m}$ and $d / \Lambda=0.53$ for LCF7, $\Lambda=12.0 \mu \mathrm{m}$ and $d / \Lambda=0.48$ for LCF19, and $\Lambda=8.9 \mu \mathrm{m}$ and $d / \Lambda=0.446$ for LCF37. Figure 3 (a) shows the numerically calculated bending losses of the fundamental mode at 1064-nm wavelength as a function of the bending radius in four different LCFs structures; LCF1 $(\Lambda=41.9 \mu \mathrm{m}, d / \Lambda=0.68)$, LCF7, LCF19, and LCF37. We employed a full-vector FEM in a local cylindrical 
coordinate system [6] for efficient and accurate analysis of bending losses in curved LCFs. Since these four LCFs have the same effective mode area of $1400 \mu \mathrm{m}^{2}$ and the same level of leakage loss of $1 \mathrm{~dB} / \mathrm{m}$ for the second-order mode at $1064 \mathrm{~nm}$, we could expect that these four different LCFs would show similar macro-bending loss characteristics. Surprisingly, the newly proposed LCF7, LCF19, and LCF37 show totally different bending losses and LCF37 can achieve much lower bending loss compared with LCF1. It can be seen that the allowable bending radius decreases with an increase in the number of rods in the core. This is because a gap between the air holes, which is defined as $l=\Lambda-d$, decreases with an increase of the number of rods in the core. The value of $l$ is an important parameter regarding the bending loss of the fundamental mode and it should be as small as possible, however, further increment of the number of rods in core can not provide better performance due to an increase of leakage loss of the fundamental mode. In Figs. 3 (b) and (c), we plot the optical field distributions in the curved LCF1 and LCF37, respectively, where the operating wavelength is $1064 \mathrm{~nm}$ and the bending radius is $15 \mathrm{~cm}$. As we can see, the optical field spreads out into the outer cladding of LCF1, whereas on the other hand the field is still confined into the solid core of LCF37.

\section{Conclusion}

We have described a novel methodology for realizing an effectively singlemode LCF with LMA and low bending losses. Numerical simulations carried out through full-vector FEM have revealed that the proposed solid core LCFs formed by multiple unit-cells area can achieve much lower bending losses in comparison to reported LCF with six large air-holes surrounding the core [4].

\section{Acknowledgments}

K. Saitoh would like to acknowledge the Strategic International Cooperative Program of Japan Science and Technology Agency (JST) for supporting a part of this work. 